\title{
Glutamic acid decarboxylase antibodies are indicators of the course, but not of the on- set, of diabetes in middle-aged adults: the Atherosclerosis Risk in Communities Study
}

A. Vigo ${ }^{1}$, B.B. Duncan ${ }^{1,2}$, M.I. Schmidt ${ }^{1,2}$, D. Couper ${ }^{3}$,

G. Heiss ${ }^{2}$, J.S. Pankow ${ }^{4}$ and C.M. Ballantyne ${ }^{5}$
1 Programa de Pós-Graduação em Epidemiologia, Faculdade de Medicina, Universidade Federal do Rio Grande do Sul, Porto Alegre, RS, Brasil ${ }^{2}$ Department of Epidemiology, ${ }^{3}$ Department of Biostatistics, School of Public Health at Chapel Hill, University of North Carolina, Chapel Hill, NC, USA ${ }^{4}$ Division of Epidemiology, School of Public Health, University of Minnesota, Minneapolis, MN, USA

${ }^{5}$ Department of Medicine, Baylor College of Medicine, Houston, TX, USA

\author{
Correspondence \\ A. Vigo \\ Programa de Pós-Graduação em \\ Epidemiologia \\ Faculdade de Medicina, UFRGS \\ Rua Ramiro Barcelos, 2600/414 \\ 90035-003 Porto Alegre, RS \\ Brasil \\ Fax: +55-51-3308-5306 \\ E-mail: alvaro.vigo@ufrgs.br \\ Part of this manuscript was \\ presented as a poster at the \\ American Diabetes Association \\ 66th Annual Scientific Sessions, \\ Washington, DC, and as part of a \\ Contributed Paper Oral Presentation \\ Session at the 2nd North American \\ Congress of Epidemiology, June \\ 21-24, 2006, Seattle, WA, USA. \\ Research supported by the National \\ Institute of Diabetes, Digestive and \\ Kidney Diseases (Grant R01-DK56918), \\ and National Heart, Lung, and Blood \\ Institute Contracts (N01-HC-55015, \\ -55016, -55018, -55019, -55020, \\ -55021 , and -55022). B.B. Duncan \\ and M.I. Schmidt received support \\ from the Centers of Excellence Grant \\ of CNPq.
}

Received October 30, 2006 Accepted April 13, 2007

\begin{abstract}
To efficiently examine the association of glutamic acid decarboxylase antibody (GADA) positivity with the onset and progression of diabetes in middle-aged adults, we performed a case-cohort study representing the $\sim 9$-year experience of 10,275 Atherosclerosis Risk in Communities Study participants, initially aged 45-64 years. Antibodies to glutamic acid decarboxylase $\left(\mathrm{GAD}_{65}\right)$ were measured by radioimmunoassay in 580 incident diabetes cases and 544 non-cases. The overall weighted prevalence of GADA positivity $(\geq 1 \mathrm{U} / \mathrm{mL})$ was $7.3 \%$. Baseline risk factors, with the exception of smoking and interleukin-6 ( $\mathrm{P} \leq 0.02)$, were generally similar between GADApositive and -negative individuals. GADA positivity did not predict incident diabetes in multiply adjusted $(\mathrm{HR}=1.04 ; 95 \% \mathrm{CI}=0.55$, 1.96) proportional hazard analyses. However, a small non-significant adjusted risk $(\mathrm{HR}=1.29 ; 95 \% \mathrm{CI}=0.58,2.88)$ was seen for those in the highest tertile $(\geq 2.38 \mathrm{U} / \mathrm{mL})$ of positivity. GADA-positive and GADA-negative non-diabetic individuals had similar risk profiles for diabetes, with central obesity and elevated inflammation markers, aside from glucose, being the main predictors. Among diabetes cases at study's end, progression to insulin treatment increased monotonically as a function of baseline GADA level. Overall, being GADA positive increased risk of progression to insulin use almost 10 times $(\mathrm{HR}=9.9 ; 95 \% \mathrm{CI}=3.4,28.5)$. In conclusion, in initially non-diabetic middle-aged adults, GADA positivity did not increase diabetes risk, and the overall baseline profile of risk factors was similar for positive and negative individuals. Among middle-aged adults, with the possible exception of those with the highest GADA levels, autoimmune pathophysiology reflected by GADA may become clinically relevant only after diabetes onset.
\end{abstract}

Key words - Diabetes mellitus

- Glutamic acid decarboxylase

- Auto-immune diseases

- Inflammation

- Risk factors 


\section{Introduction}

Glutamic acid decarboxylase antibodies (GADA) are present in most patients with autoimmune diabetes at or before diagnosis and are related to insulin secretion abnormalities and to the onset of type 1 diabetes (1). Reported to be present in $<5 \%$ to more than $20 \%$ of adults diagnosed as presumably having type 2 diabetes (2-7), GADA are also markers of a slowly evolving form of autoimmune diabetes, frequently called latent autoimmune diabetes in adults (LADA) $(4,8,9)$. Most studies to date have evaluated individuals at or after diagnosis of diabetes, and little attention has been directed to the question of whether the presence of GADA predicts the development of diabetes in middle-aged adults.

Subclinical inflammation has been found to predict the development of diabetes with onset in middle age (10). Although this association is in great part related to obesity, it is logical to inquire whether it also reflects the fact that some incident, middle-aged cases may have a subclinical, chronic autoimmune disease affecting $\beta$ cells.

The objectives of the present study were to characterize the importance of GADA positivity in a representative sample of initially non-diabetic middle-aged individuals enrolled in the Atherosclerosis Risk in Communities (ARIC) Study. Specifically, we aimed to describe the frequency and correlates of GADA positivity, to evaluate whether GADA positivity predicts the development of diabetes, and to evaluate whether risk factors (including inflammation markers) for diabetes and the early clinical course of diabetes are similar in GADA-positive and GADA-negative individuals.

\section{Subjects and Methods}

In 1987-89 the ARIC Study recruited a population-based cohort of 15,792 men and women 45 to 64 years of age from four US communities (11). All subjects were invited to return to three clinic visits, at approximately 3-year intervals, at which incident diabetes was ascertained. Human subjects research review committees at the involved institutions approved the study, and all participants gave written informed consent.

In order to permit an efficient use of ARIC frozen biologic specimens, a casecohort design was chosen to investigate our objectives. All specimens were stored at $-70^{\circ} \mathrm{C}$ within $90 \mathrm{~min}$ of venipuncture. Before sampling, we excluded 2018 participants with prevalent diabetes, 95 members of minority ethnic groups with small numbers, 853 who did not return to any follow-up visit, 26 having no valid diabetes determination at follow-ups, 7 with restrictions on stored plasma use, 12 with missing baseline anthropometrics, and 2506 participants in previous ARIC case-control studies involving cardiovascular disease for whom stored plasma was either previously exhausted or held in reserve. This resulted in a final sample of 10,275 individuals ( $75 \%$ of those in the full cohort without diabetes at baseline), 1155 of whom (11.2\%) developed diabetes during follow-up. From these 10,275 eligible members of the cohort, we selected and measured analytes on ethnicity-stratified random samples of cases of incident diabetes and of eligible members of the full cohort (a total of 1198 individuals). A few of the incident cases of diabetes overlapped with the cohort sample, and a few were selected only via the cohort sample. Of those sampled, we excluded 45 for incomplete fasting $(<8 \mathrm{~h})$ or for not having values for all covariates, 27 identified as non-cases but having 2 -h glycemia $\geq 11.1 \mathrm{mmol} / \mathrm{L}$ during the oral glucose tolerance test performed at the last follow-up visit, and 2 individuals lacking information about antibodies for GADA, leaving a total of 1124 subjects for analysis.

At baseline and at follow-up visits, glucose was measured by a hexokinase method, 
while fasting serum insulin was measured by a nonspecific radioimmunoassay. The cross-reactivity with pro-insulin was $33 \%$. Waist girth was measured at the umbilical level, and hip circumference at the maximum hip girth. The waist-to-hip ratio (WHR) was defined as the ratio between these two measurements. A family history of diabetes was defined as a report of diabetes in either parent. The definitions and methods for other baseline measurements (height, weight, smoking status, systolic blood pressure, triglycerides, HDL cholesterol, insulin, white cell count, and fibrinogen) have been previously reported (12).

Antibodies to glutamic acid decarboxylase $\left(\mathrm{GAD}_{65}\right)$ were measured in duplicate by radioimmunoassay using human recombinant $\mathrm{I}^{125}$ according to the manufacturer's protocol (Kronus, Boise, ID, USA). The mean of duplicate measurements was used. The reliability coefficient (the ratio of betweenperson to total variance obtained by analyzing replicate pairs of baseline samples drawn at one sitting from a subset of subjects) was 0.98. Subjects were considered positive for GADA if the value was $1 \mathrm{U} / \mathrm{mL}$ or higher.

The acute-phase markers C-reactive protein (CRP), orosomucoid, sialic acid, and fibrinogen, as well as interleukin (IL)-6 and white cell count, were chosen to characterize chronic systemic inflammation. White cell count was determined at each center with Coulter counters. The remaining determinations were performed at central laboratories: fibrinogen at baseline in citrated samples using thrombin-time titration, and the other analytes on plasma specimens frozen at baseline. IL-6 levels were measured by high-sensitivity enzyme-linked immunosorbent assay (ELISA; R\&D systems, Minneapolis, MN, USA), sialic acid by enzymatic determination using a colorimetric assay (13) (Roche Diagnostics, Indianapolis, IN, USA), orosomucoid utilizing an immunoturbidimetric technique (Kamiya Biomedical, Seattle, WA, USA), and high-sensitivity
CRP (hsCRP - Latex II, Denka Seikan, Tokyo, Japan) on a Hitachi 911 automated analyzer (Indianapolis, IN, USA). An additional analyte, leptin, was measured in duplicate by direct sandwich ELISA (Linco Research, Inc., St. Charles, MO, USA) on the case cohort sample.

As in previous reports $(14,15)$, we created a score to indicate low-grade systemic inflammation ranging from 0 to 6 , attributing 1 point to a value greater than the median of the cohort sample for each of the six inflammation markers - IL-6, CRP, orosomucoid, sialic acid, white cell count, and fibrinogen.

Diabetes was defined on the basis of 1) a reported physician diagnosis, 2) use of antidiabetes medications, 3) a fasting ( $\geq 8 \mathrm{~h}$ ) glucose $\geq 7.0 \mathrm{mmol} / \mathrm{L}$, or 4 ) a non-fasting glucose of $\geq 11.1 \mathrm{mmol} / \mathrm{L}$. For incident diabetes ascertained on the basis of a glucose value, incident date was estimated by linear interpolation using glucose values at the ascertaining visit and the previous one. The fasting glucose at ascertainment for subjects who had been told they had diabetes or who were on diabetic medication may have been affected by their knowledge of their diabetic status (and in some cases was even below $7.0 \mathrm{mmol} / \mathrm{L}$ ). For these subjects, the time to reach $7.0 \mathrm{mmol} / \mathrm{L}$ was estimated by using their fasting glucose at the earlier visit and a slope estimated using information from all diabetic subjects who had been unaware of their status. Hypertension was defined as a blood pressure $\geq 140 / 90 \mathrm{mmHg}$ or reported use of anti-hypertensive medication.

\section{Statistical analysis}

Statistical analysis was based on our casecohort sampling design, weights being defined as the inverse of the ethnicity-specific sampling fractions, thus permitting statistical estimation and inference relevant to the entire cohort. In the cohort random sample, we did weighted analysis of covariance to 
compute adjusted means and proportions of socio-demographic variables and risk factors. Tertiles of GADA levels among GADApositive subjects, used in some of the analyses, were computed in the cohort random sample. We tested differences in clinical characteristics adjusted for baseline levels using a similar weighted covariance analysis. Unadjusted and adjusted relative risks of developing diabetes and of progressing to insulin use once diabetes was present were estimated in proportional hazards models fitted using SUDAAN to account for the case-cohort design with stratified sampling both from the whole cohort and from the incident diabetes cases (16). Statistical significance of differences in hazard ratios between GADA-positive and -negative individuals was evaluated by Wald tests of the interaction terms in similar models. Analyses were performed using SAS (17) and SUDAAN (18).

\section{Results}

After the exclusions, the cohort random sample comprised 639 individuals and the incident diabetes random sample 558 subjects. With overlap, the total sample consisted of 1124 subjects, 580 who went on to develop incident diabetes and 544 who did not. The median (interquartile range) follow-up to time of onset in those who developed diabetes was 3.0 (1.7-5.9) years and to censoring for those who did not was 8.9 (8.7-9.0) years. Of the incident case subjects, $498(86 \%)$ were ascertained by a fasting glucose $\geq 7.0 \mathrm{mmol} / \mathrm{L}, 5(1 \%)$ by a nonfasting glucose $\geq 11.1 \mathrm{mmol} / \mathrm{L}$, and, independently of their glucose values, 125 (22\%) by reported physician diagnosis or medication use. The characteristics of this case-cohort sample have been previously described (14). Among GADA-positive and -negative participants, 38.6 and $37.3 \%$ were male, 32.3 and $21.7 \%$ had parental history of diabetes, and 30.2 and $22.0 \%$ were African-
American, respectively. Mean \pm SEM ages were $51.6 \pm 0.66$ and $52.6 \pm 0.23$ years and mean body mass index (BMI) were $27.2 \pm$ 0.80 and $27.3 \pm 0.21 \mathrm{~kg} / \mathrm{m}^{2}$, respectively. None of these differences was statistically significant $(\mathrm{P}>0.05)$.

For cases who returned to the final ARIC clinic visit, weighted mean $(95 \% \mathrm{CI})$ followup after estimated date of diabetes onset was 4.8 (3.8-5.8) years for those who were GADA positive and 4.9 (4.7-5.2) for those who were GADA negative.

Among the subjects of the cohort random sample, 52 were GADA positive and 587 were GADA negative, the weighted prevalence of GADA positivity being $7.3 \%$. Among those who went on to develop diabetes, $18(5.9 \%$; $95 \% \mathrm{CI}=3.3,8.6 \%)$ whites and $23(8.3 \%$; 95\% CI $=5.1,11.6 \%)$ African-Americans were positive for GADA. Among GADA-positive subjects, most (55\%) had low-antibody levels $(<2 \mathrm{U} / \mathrm{mL})$, and only $10 \%$ had values greater than $10 \mathrm{U} /$ $\mathrm{mL}$.

Few differences in clinical characteristics were seen according to the presence of GADA among the participants of the cohort random sample. Current smoking was less common among GADA-positive individuals $(\mathrm{P}=0.02)$. Among the markers of inflammation, only IL-6 showed a significant difference, being slightly lower among GADApositive individuals $(\mathrm{P}=0.01)$. However, when inflammation was assessed by an aggregate score, no difference was apparent, with an inflammation score (mean \pm SEM) of $2.6 \pm 0.24$ for GADA-positive subjects $v s$ $3.0 \pm 0.08$ for GADA-negative subjects $(\mathrm{P}=$ $0.15)$. Statistical significance of GADA-inflammation marker associations was unchanged after adjusting for differences in smoking prevalence. Additionally, no differences were seen between GADA-positive and -negative individuals in age, gender, race, family history of diabetes, prevalence of hypertension, obesity indices, fasting insulin, glucose, homa insulin sensitivity or 
beta cell function, triglycerides, HDL cholesterol, leptin, or adiponectin.

To further examine differences in clinical characteristics according to GADA levels, within the limits imposed by a sample of 84 GADA-positive individuals, we compared these characteristics across tertiles of GADApositive individuals, with the cutoff points for these tertiles being 1.37 and $2.38 \mathrm{U} / \mathrm{mL}$. Though no difference was seen in obesity measures overall, those with GADA values in the highest tertile had somewhat lower BMI, $26.1 \pm 1.23 \mathrm{~kg} / \mathrm{m}^{2}$, and waist circumference, $92.4 \pm 3.77 \mathrm{~cm}$, than GADA-negative subjects $\left(27.3 \pm 0.21 \mathrm{~kg} / \mathrm{m}^{2}\right.$ and $94.9 \pm$ $0.53 \mathrm{~cm}, \mathrm{P}=0.35$ and 0.55 , respectively). Lowest values for IL-6 $(2.0 \pm 0.22 \mathrm{pg} / \mathrm{mL})$ and the inflammation score $(2.5 \pm 0.38)$ were seen in the 3rd GADA tertile. White cell count $\left(5.3 \pm 0.25 v s 5.9 \pm 0.03 \times 10^{9} / \mathrm{L}\right.$ in GADA negatives) and orosomucoid (78.2 \pm 3.85 vs $87.3 \pm 0.84 \mathrm{mg} / \mathrm{dL}$ in GADA negatives) were also notably lower in this tertile.

Next we evaluated the association of GADA with the development of diabetes using proportional hazard models. The presence of GADA was not associated with the development of diabetes in unadjusted analyses $(\mathrm{HR}=0.93 ; 95 \% \mathrm{CI}=0.59,1.48)$, nor when adjusted for age, gender, ethnicity, family history of diabetes, glucose, BMI, WHR, insulin, hypertension, and inflammation score ( $\mathrm{HR}=1.04 ; 95 \% \mathrm{CI}=0.55,1.96)$. The association was also absent, when analyzed separately, in men and women, blacks and whites, younger and older subjects (45-54 and 5564 years), and smokers and non-smokers. GADA-positive lean individuals (BMI $<25$ $\mathrm{kg} / \mathrm{m}^{2}$ ) had a somewhat larger, but not statistically significant risk of developing diabetes compared to GADA-negative lean individuals $(\mathrm{HR}=1.23 ; 95 \% \mathrm{CI}=0.47,3.25)$. Individuals in the highest tertile of GADA ( $\sim 3 \%$ of the cohort random sample) had no greater risk in crude analyses $(\mathrm{HR}=1.08$, $95 \% \mathrm{CI}=0.21,5.49)$ nor in the fully adjusted model $(\mathrm{HR}=1.45 ; 95 \% \mathrm{CI}=0.39,5.42)$.
To compare the risk factor profile for developing diabetes with and without GADA, we next modeled incident diabetes separately for GADA-positive and GADA-negative individuals. Table 1 (top) shows that the magnitude of risk is generally similar for each group in univariate analyses, the inflammation score being the only variable demonstrating greater risk to GADA-positive individuals. Since there were only 40 GADA-positive cases, we performed multivariable modeling starting with all available risk factors for diabetes and using backward elimination until 4 variables remained, 3 being statistically significant (WHR, baseline fasting glucose, and inflammation score, Table 1, bottom). Modeling with these same

Table 1. Crude and adjusted associations of risk factors for incident diabetes according to glutamic acid decarboxylase antibody (GADA) positivity, Atherosclerosis Risk in Communities Study, 1987-98.

\begin{tabular}{|c|c|c|c|c|c|c|}
\hline \multirow[t]{2}{*}{ Risk factors } & \multirow[t]{2}{*}{$\begin{array}{l}\text { Standard } \\
\text { deviation }\end{array}$} & \multicolumn{2}{|c|}{$\begin{array}{c}\text { GADA positive } \\
(\mathrm{N}=84)\end{array}$} & \multicolumn{2}{|c|}{$\begin{array}{l}\text { GADA negative } \\
\quad(N=1040)\end{array}$} & \multirow[t]{2}{*}{$\begin{array}{l}{ }^{*} \mathrm{P} \\
\text { value }\end{array}$} \\
\hline & & $\mathrm{HR}$ & $95 \% \mathrm{Cl}$ & $\mathrm{HR}$ & $95 \% \mathrm{Cl}$ & \\
\hline \multicolumn{7}{|l|}{ Crude associations } \\
\hline Age (years) & 5.41 & 1.67 & $1.07 ; 2.60$ & 1.17 & $1.04 ; 1.32$ & 0.15 \\
\hline Body mass index $\left(\mathrm{kg} / \mathrm{m}^{2}\right)$ & 5.64 & 1.57 & $1.03 ; 2.38$ & 1.82 & $1.55 ; 2.13$ & 0.53 \\
\hline Waist-to-hip ratio & 0.08 & 3.07 & $1.69 ; 5.57$ & 1.83 & $1.54 ; 2.19$ & 0.12 \\
\hline Insulin $(\mathrm{pmol} / \mathrm{L})^{\mathrm{a}}$ & 0.30 & 2.59 & $1.34 ; 4.99$ & 3.18 & $2.71 ; 3.74$ & 0.57 \\
\hline Male & & 1.24 & $0.50 ; 3.12$ & 1.25 & $0.97 ; 1.62$ & 0.97 \\
\hline African-American & & 2.00 & $0.91 ; 4.41$ & 2.12 & $1.98 ; 2.28$ & 0.99 \\
\hline Family history of diabetes & & 1.43 & $0.56 ; 3.67$ & 1.92 & $1.45 ; 2.54$ & 0.60 \\
\hline Hypertension & & 2.71 & $1.08 ; 6.85$ & 2.54 & $1.97 ; 3.28$ & 0.86 \\
\hline Current smoker & & 1.55 & $0.46 ; 5.23$ & 0.90 & $0.68 ; 1.21$ & 0.35 \\
\hline Glucose (mmol/L) & 0.51 & 4.51 & $2.73 ; 7.44$ & 3.46 & $2.91 ; 4.12$ & 0.36 \\
\hline Leptin & & 2.24 & $0.91 ; 5.54$ & 1.69 & $1.32 ; 2.17$ & 0.52 \\
\hline Women only & & 6.26 & $1.61 ; 24.33$ & 3.08 & $2.19 ; 4.33$ & 0.31 \\
\hline Inflammation score & 1.79 & 3.05 & $1.79 ; 5.20$ & 1.73 & $1.52 ; 1.96$ & 0.04 \\
\hline \multicolumn{7}{|l|}{ Adjusted associations } \\
\hline Waist-to-hip ratio & 0.08 & 2.20 & $1.43 ; 3.38$ & 1.32 & $1.10 ; 1.58$ & 0.03 \\
\hline Hypertension & & 0.36 & $0.13 ; 1.00$ & 1.00 & $0.71 ; 1.42$ & 0.38 \\
\hline Glucose (mmol/L) & 0.51 & 4.33 & $2.58 ; 7.27$ & 3.09 & $2.54 ; 3.76$ & 0.30 \\
\hline Inflammation score & 1.79 & 1.82 & $1.06 ; 3.12$ & 1.37 & $1.15 ; 1.63$ & 0.31 \\
\hline
\end{tabular}

For continuous variables, the hazard ratio (HR) is expressed for a difference of 1 standard deviation of the variable (in the cohort random sample), with the exception of leptin, which is dichotomized as above or below the sex-specific median value. Adjusted associations were obtained by proportional hazards modeling for the four variables with smallest $P$ values in backward modeling of GADA-positive cases. ${ }^{a} \log _{10}$-transformed. *For difference in hazard ratio between GADA-positive and GADAnegative individuals. 
Figure 1. Insulin use at end of follow-up among individuals by the presence and level of glutamic acid decarboxylase antibody (GADA) positivity. risk factors for GADA-negative subjects produced smaller hazard ratios for these three principal risk factors, statistically significantly so for WHR (interaction $\mathrm{P}=0.03$ ). Having a leptin value above the sex-specific median $(4.43 \mathrm{ng} / \mathrm{mL}$ for males and $17.03 \mathrm{ng} /$ $\mathrm{mL}$ for females) was not a risk factor in GADA-positive cases, either in crude or adjusted $(\mathrm{HR}=1.54 ; 95 \% \mathrm{CI}=0.52,4.54)$ analyses. However, leptin, in crude analyses, was highly associated with developing diabetes among the 53 GADA-positive women $(\mathrm{HR}=6.26,95 \% \mathrm{CI}=1.61,24.33)$, though not statistically so after adjustment for WHR ( $\mathrm{HR}=2.57,95 \% \mathrm{CI}=0.59,11.18)$.

To compare clinical progression of GADA-positive and-negative incident cases, we determined case characteristics at the last

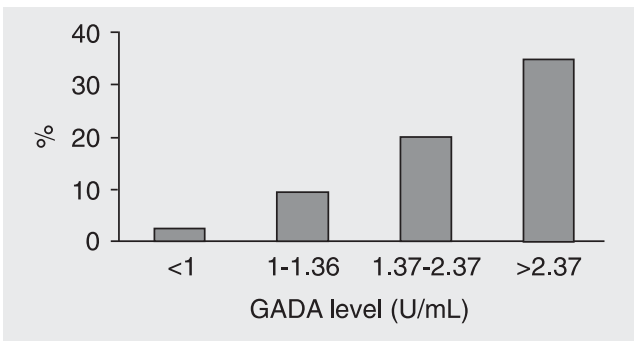

ARIC follow-up visit (Table 2). Among ascertained cases of diabetes present at this final visit, a somewhat greater percentage of GADA-positive (59\%) than GADA-negative (49\%) subjects reported having received a clinical diagnosis of diabetes (or to be currently using anti-diabetes medication) ( $\mathrm{P}$ $=0.32$ ). Anti-diabetes medication use was reported by $30 \%$ of GADA-positive and $28 \%$ of GADA-negative cases. However, among medication users at visit $4,62 \%$ of those GADA positive $v s$ only $9 \%$ of those GADA negative were using insulin. Thus, GADA positivity notably increased risk of progressing to insulin use once diabetes was present $(\mathrm{HR}=9.9 ; 95 \% \mathrm{CI}=3.4,28.5 ; \mathrm{P}<0.001)$. Furthermore, progression to insulin treatment increased with greater baseline GADA values $(9.1,20.0$, and $34.6 \%$ for those in the 1st, 2nd and 3rd GADA tertiles, vs $2.4 \%$ in those GADA negative; Figure 1).

Additional characteristics of GADA-positive and -negative cases at visit 4 are presented in Table 2. Comparison of means across all categories revealed no statistically significant differences overall, perhaps due to the very small size of GADA tertile case

\begin{tabular}{|c|c|c|c|c|c|}
\hline Variable & $\begin{array}{c}\text { GADA } \\
\text { negative } \\
(\mathrm{N}=450)\end{array}$ & $\begin{array}{c}\text { GADA positive } \\
\text { 1st tertile } \\
(\mathrm{N}=14)\end{array}$ & $\begin{array}{l}\text { GADA positive } \\
\text { 2nd tertile } \\
(\mathrm{N}=10)\end{array}$ & $\begin{array}{l}\text { GADA positive } \\
\text { 3rd tertile } \\
(\mathrm{N}=10)\end{array}$ & ${ }^{\star} \mathrm{P}$ \\
\hline \multicolumn{6}{|l|}{ Waist $(\mathrm{cm})$} \\
\hline At follow-upa & $110.4 \pm 0.70$ & $114.4 \pm 4.94$ & $101.8 \pm 2.93$ & $99.6 \pm 4.98$ & 0.41 \\
\hline Difference from baseline & $5.6 \pm 0.38$ & $6.6 \pm 3.18$ & $6.5 \pm 2.40$ & $1.8 \pm 3.19$ & 0.53 \\
\hline \multicolumn{6}{|l|}{ Weight (kg) } \\
\hline At follow-upa & $90.2 \pm 0.85$ & $95.0 \pm 6.08$ & $76.6 \pm 4.31$ & $82.3 \pm 4.54$ & 0.63 \\
\hline Difference from baseline & $3.0 \pm 0.38$ & $3.6 \pm 3.39$ & $2.5 \pm 2.74$ & $-0.16 \pm 1.43$ & 0.69 \\
\hline \multicolumn{6}{|l|}{ BMI $\left(\mathrm{kg} / \mathrm{m}^{2}\right)$} \\
\hline At follow-upa & $32.1 \pm 0.29$ & $33.1 \pm 1.94$ & $28.9 \pm 1.28$ & $29.4 \pm 1.28$ & 0.58 \\
\hline Difference from baseline & $1.3 \pm 0.14$ & $1.4 \pm 1.16$ & $1.2 \pm 1.09$ & $0.04 \pm 0.49$ & 0.63 \\
\hline
\end{tabular}

Data are reported as means \pm SEM. BMI $=$ body mass index. Cutoff points for GADA tertiles were 1.37 and 2.38 .

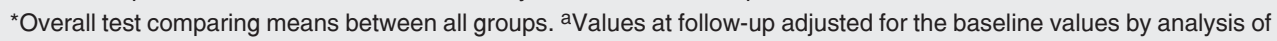
covariance. 
groups. Nonetheless, at study conclusion, those in the highest tertile $(\geq 2.38 \mathrm{U} / \mathrm{mL})$ of GADA at baseline had waist girth $11 \mathrm{~cm}$ smaller and BMI $2.7 \mathrm{~kg} / \mathrm{m}^{2}$ less than GADAnegative individuals. Gains in waist and weight from baseline were likewise $4 \mathrm{~cm}$ and $3 \mathrm{~kg}$ less for those in the highest tertile of GADA. Similar differences in final anthropometric values were seen among those with GADA values between 1.37 and $2.38 \mathrm{U} / \mathrm{mL}$, but not for those with GADA values less than $1.37 \mathrm{U} / \mathrm{mL}$. Among cases not reporting medication use, GADA-positive and GADAnegative individuals had virtually identical values for fasting and 2-h glucose, fasting insulin, triglycerides, and HDL cholesterol (data not shown).

\section{Discussion}

Our results demonstrate that approximately $7 \%$ of middle-aged ARIC participants were positive for GADA, a value similar to those of previous population-based reports. Those positive and negative had similar levels of most diabetes risk factors. Although a non-significant tendency toward increased risk was seen with highest levels of GADA, antibody positivity was not a risk factor for the development of diabetes among ARIC Study participants. Inflammation, central obesity and high baseline glucose, known to predict type 2 diabetes, were the principal diabetes risk factors for both GADA-positive and -negative cases. Important differences in obesity measures and insulin use were found in diabetes cases with higher GADA levels estimated 4.8 years after ascertainment, a monotonic increase in insulin use being seen with increasing baseline GADA levels.

We know of no other published study of associations with GADA status in initially non-diabetic subjects. Previously published studies, including population-based ones (19) describe associations with already prevalent diabetes. Two groups, the Diabetes Preven- tion Project (DPP) (20) and the Botnia Study (21), have recently reported in abstract form that autoantibodies do predict incident diabetes. However both, like our study, did not find an association with a single measurement of GADA. In the DPP, the association was largely explained by the presence, considerably less frequent, of the IA-2 antibody. In the Botnia Study, an association was seen only in younger $(<45$ years old $)$ subjects with repeated positivity $(\sim 1 / 3$ of all those positive) or in those in the upper quartile of GADA positivity. Both the DPP and the Botnia Study extended enrollment to younger participants than did ARIC. Neither presented data exclusively for middle-aged and elderly individuals, age ranges in which the bulk of diabetes cases are detected.

Given what is currently believed about the autoimmune origin of LADA, the relative similarity between GADA-positive and -negative subjects at ARIC baseline, when coupled with absence of risk associated with GADA positivity, is surprising. Previous studies, such as that of the UK Prospective Diabetes Study (UKPDS) (22), have suggested that GADA positivity identifies a subtype of adult onset diabetes of distinct etiology, affecting leaner subjects with less pancreatic reserve than those with type 2 diabetes. Here too, differences in study populations may explain the apparent discrepancies. The UKPDS enrolled patients aged between 25 and 65 years shortly after clinical diagnosis. By contrast, ARIC incident cases were all over 45 years, only $22 \%$ being ascertained because of clinical diagnosis, and only $59 \%$ of GADA-positive cases being clinically diagnosed by the end of follow-up. If one assumes that 10 years is the average lag between the onset of diabetes and its clinical diagnosis, and that some additional lag existed between clinical diagnosis and enrollment in the UKPDS, our GADA-positive cases were ascertained, as a group, more than 5 years earlier in the natural history of their disease than those of the 
UKPDS. In fact, in the UKPDS, a study of much greater size, differences in the clinical profile among older ( $>55$ years of age) GADA-positive and -negative patients were less than among younger ones (22). The fact that GADA-positive subjects in ARIC did not have higher levels of inflammation markers provides further evidence that an autoimmune pancreatitis is not the source of subclinical inflammation characteristic of the pre-diabetic state in adults. That central obesity and subclinical inflammation were important risk factors for diabetes onset for these individuals suggests a pathophysiology for GADA-positive cases quite similar to that of type 2 diabetes. In the majority of GADA-positive individuals who developed diabetes, the autoimmune pancreatitis denoted by GADA, instead of precipitating disease onset, may rather lead to a more rapid pancreatic failure once islet cells are stressed by the diabetic milieu. Indeed, our study, like others, clearly suggests a rapid progression to insulin use in a large fraction of GADA-positive ( $>1 \mathrm{U} / \mathrm{mL}$ ) cases.

In this regard, leptin has been suggested in an animal study (23) to accelerate autoimmune diabetes in females. Though crude analysis showed a large risk for high-leptin values in women, our sample of GADApositive women is too small to permit adequate multivariable investigation of this complex issue.

The lower prevalence of smoking seen in GADA-positive individuals at baseline may be due to random error, as multiple comparisons were made and $\mathrm{P}$ values were not far from borderline significance. However, nicotine, whose plasma levels are increased in smokers, has been reported to have anti-inflammatory actions in macrophages and adipocytes (24-26). Its anti-inflammatory effects might protect against autoimmune pancreatitis. In this regard, others have reported that smoking protects against the development of GADA-positive diabetes. Compared with adult Norwegian subjects who had never smoked, the adjusted risk of developing LADA $(\mathrm{N}=$ $81 ; \mathrm{RR}=0.25 ; 95 \% \mathrm{CI}=0.11,0.60)$ or type 1 diabetes $(\mathrm{N}=18 ; \mathrm{RR}=0.17 ; 95 \% \mathrm{CI}=0.04$, 0.73 ), was substantially lower for current $v$ s never smokers, whereas the adjusted risk of developing type 2 diabetes was greater $(\mathrm{N}=$ $738 ; \mathrm{RR}=1.64 ; 95 \% \mathrm{CI}=1.12,2.39$ ) for current heavy $v s$ never smokers (27). Our data suggest a possible alternative explanation for this reported association - that smoking, by furnishing nicotine, diminishes or abolishes the autoimmune state which leads to GADA positivity, without protecting against the development of diabetes in those who remain GADA positive. Smoking has been repeatedly suggested, if anything, to be a diabetes risk factor (27-29).

Limitations to our study should be noted. The number of GADA-positive individuals and GADA-positive cases of incident diabetes in our sample was relatively small, limiting our ability to describe and contrast clinical characteristics and to perform multivariable modeling. We did not measure additional autoantibodies. In terms of the generalizability of our findings, our GADA-positive cases were considerably obese, as indicated by their waist girth, as a group, of $106.5 \mathrm{~cm}$ at the end of follow-up. GADA positivity could possibly be more important in the development of diabetes in middleaged or elderly populations with less obesity than our ARIC sample. Additional studies on the early natural history of diabetes in middle-aged and elderly GADA-positive individuals are warranted.

In conclusion, in these middle-aged adults, GADA positivity did not increase diabetes risk, and the overall profile of risk factors was similar for positive and negative individuals. These ARIC data suggest that, with the possible exception of those with highest GADA levels, the presumably lowgrade pancreatitis reflected specifically by GADA appears to become clinically relevant in middle-aged individuals only after diabetes onset, with more rapid progression 
to insulin use. Additional prospective studies, including a wider range of antibodies when possible, are necessary to clarify the role of autoimmunity in the onset of diabetes in the middle-aged and elderly.

\section{Acknowledgments}

The authors thank the staff and participants in the ARIC Study for their important contributions.

\section{References}

1. Jun HS, Khil LY, Yoon JW. Role of glutamic acid decarboxylase in the pathogenesis of type 1 diabetes. Cell Mol Life Sci 2002; 59: 1892-1901.

2. Zimmet PZ, Tuomi T, Mackay IR, Rowley MJ, Knowles W, Cohen M, et al. Latent autoimmune diabetes mellitus in adults (LADA): the role of antibodies to glutamic acid decarboxylase in diagnosis and prediction of insulin dependency. Diabet Med 1994; 11: 299-303.

3. Naik RG, Palmer JP. Latent autoimmune diabetes in adults (LADA). Rev Endocr Metab Disord 2003; 4: 233-241.

4. Pozzilli $P$, Di Mario U. Autoimmune diabetes not requiring insulin at diagnosis (latent autoimmune diabetes of the adult): definition, characterization, and potential prevention. Diabetes Care 2001; 24: 14601467.

5. Zimmet PZ, Elliott RB, Mackay IR, Tuomi T, Rowley MJ, Pilcher CC, et al. Autoantibodies to glutamic acid decarboxylase and insulin in islet cell antibody positive presymptomatic type 1 diabetes mellitus: frequency and segregation by age and gender. Diabet Med 1994; 11: 866-871.

6. Zimmet PZ. The pathogenesis and prevention of diabetes in adults. Genes, autoimmunity, and demography. Diabetes Care 1995; 18: 1050-1064.

7. Zimmet $P$, Turner R, McCarty D, Rowley M, Mackay I. Crucial points at diagnosis. Type 2 diabetes or slow type 1 diabetes. Diabetes Care 1999; 22 (Suppl 2): B59-B64.

8. Fourlanos S, Dotta F, Greenbaum CJ, Palmer JP, Rolandsson O, Colman PG, et al. Latent autoimmune diabetes in adults (LADA) should be less latent. Diabetologia 2005; 48: 2206-2212.

9. Gale EA. Latent autoimmune diabetes in adults: a guide for the perplexed. Diabetologia 2005; 48: 2195-2199.

10. Duncan BB, Schmidt MI. The epidemiology of low-grade chronic systemic inflammation and type 2 diabetes. Diabetes Technol Ther 2006; 8: 7-17.

11. The Atherosclerosis Risk in Communities (ARIC) Study: design and objectives. The ARIC investigators. Am J Epidemiol 1989; 129: 687702.

12. Schmidt MI, Duncan BB, Sharrett AR, Lindberg G, Savage PJ, Offenbacher $S$, et al. Markers of inflammation and prediction of diabetes mellitus in adults (Atherosclerosis Risk in Communities Study): a cohort study. Lancet 1999; 353: 1649-1652.

13. Sugahara $\mathrm{K}$, Sugimoto $\mathrm{K}$, Nomura $\mathrm{O}$, Usui T. Enzymatic assay of serum sialic acid. Clin Chim Acta 1980; 108: 493-498.

14. Duncan BB, Schmidt MI, Pankow JS, Ballantyne CM, Couper D, Vigo $A$, et al. Low-grade systemic inflammation and the development of type 2 diabetes: the Atherosclerosis Risk in Communities Study. Diabetes 2003; 52: 1799-1805.

15. Duncan BB, Schmidt MI, Pankow JS, Bang H, Couper D, Ballantyne $\mathrm{CM}$, et al. Adiponectin and the development of type 2 diabetes: the
Atherosclerosis Risk in Communities Study. Diabetes 2004; 53: 2473-2478.

16. Lin DY. On fitting Cox's proportional hazards models to survey data. Biometrika 2000; 87: 37-47.

17. SAS Institute Inc. SAS/STAT Users Guide, Version 8. Cary: SAS Institute Inc.; 1999.

18. Shah BV, Branwell G, Bieler GS. The SUDAAN User's Manual, Release 7.5. Research Triangle Park: Research Triangle Institute; 2002.

19. Tuomi T, Carlsson A, Li H, Isomaa B, Miettinen A, Nilsson A, et al. Clinical and genetic characteristics of type 2 diabetes with and without GAD antibodies. Diabetes 1999; 48: 150-157.

20. Dabelea D, Ma Y, Knowler WC, Marcovina S, Saudek C, Arakaki R, et al. Autoimmunity and Prevention of Type 2 Diabetes: The Diabetes Prevention Program (DPP). American Diabetes Association http:// scientificsessions.diabetes.org/Abstracts/index.cfm?fuseaction= Locator.DisplayAbstractSearch\&CFID=132030\&CFTOKEN=37062675; 2006.

21. Lundgren VM, Lyssenko V, Isomaa B, Laurila E, Groop L, Tuomi T. GAD-positivity in relatives of type 2 diabetes or LADA - results from the Botnia Study. Diabetologia 2005; 48 (Suppl 1): A86 (Abstract).

22. Turner R, Stratton I, Horton V, Manley S, Zimmet P, Mackay IR, et al. UKPDS 25: autoantibodies to islet-cell cytoplasm and glutamic acid decarboxylase for prediction of insulin requirement in type 2 diabetes. UK Prospective Diabetes Study Group. Lancet 1997; 350: 12881293.

23. Matarese G, Sanna V, Lechler RI, Sarvetnick N, Fontana S, Zappacosta $S$, et al. Leptin accelerates autoimmune diabetes in female NOD mice. Diabetes 2002; 51: 1356-1361.

24. Wang H, Yu M, Ochani M, Amella CA, Tanovic M, Susarla S, et al. Nicotinic acetylcholine receptor alpha7 subunit is an essential regulator of inflammation. Nature 2003; 421: 384-388.

25. Sopori M. Effects of cigarette smoke on the immune system. Nat Rev Immunol 2002; 2: 372-377.

26. Liu RH, Kurose T, Matsukura S. Oral nicotine administration decreases tumor necrosis factor-alpha expression in fat tissues in obese rats. Metabolism 2001; 50: 79-85.

27. Carlsson S, Midthjell K, Grill V. Smoking is associated with an increased risk of type 2 diabetes but a decreased risk of autoimmune diabetes in adults: an 11-year follow-up of incidence of diabetes in the Nord-Trondelag Study. Diabetologia 2004; 47: 1953-1956.

28. Feskens EJ, Kromhout D. Cardiovascular risk factors and the 25year incidence of diabetes mellitus in middle-aged men. The Zutphen Study. Am J Epidemiol 1989; 130: 1101-1108.

29. Wannamethee SG, Shaper AG, Perry IJ. Smoking as a modifiable risk factor for type 2 diabetes in middle-aged men. Diabetes Care 2001; 24: 1590-1595. 